\title{
Magnetic Resonance Imaging of the Central Nervous System in the Kitten
}

Kazutaka YAMADA, Kazuro MIYAHARA, Motoyoshi SATO, Tsuneo HIROSE, Yukihiro YASUGI ${ }^{1)}$, Yukio MATSUDA ${ }^{1)}$, and Kazuhisa FURUHAMA ${ }^{2)}$

Department of Veterinary Clinical Radiology, Obihiro University of Agriculture and Veterinary Medicine, Obihiro, Hokkaido 080, ${ }^{1)}$ Hitachi Medical Co., Chiba 277, and ${ }^{2)}$ Daiichi Pharmaceutical Co., Tokyo 134, Japan

(Received 1 June 1994/Accepted 7 November 1994)

ABSTRACT. The optimum technical conditions for magnetic resonance imaging (MRI) of the central nervous system (CNS) in the 3-week-old kitten were investigated with a low $(0.2 \mathrm{~T})$ magnetic field MRI unit equipped with a custom-made solenoid radio frequency $(\mathrm{RF})$ coil. In the brain, $\mathrm{T} 1$ weighted images $(\mathrm{TR} / \mathrm{TE}=500 / 38 \mathrm{msec}$ ) and $\mathrm{T} 2$ weighted images (TR/TE $=1,800 / 110 \mathrm{msec}$ ) clearly delineated each anatomical portion of the brain. In the spinal cord, T2 weighted images (TR/TE $=1,500 / 110 \mathrm{msec}$ ) demonstrated structures distinctly. These results suggest that even low magnetic field MRI can be used for noninvasive scanning of small animals including new born animals.-KEY wORDS: CNS, feline, MRI.

J. Vet. Med. Sci. 57(1): 155-156, 1995

The application of magnetic resonance imaging (MRI) in the veterinary field has been increasing. Several groups of investigators have carried out imaging with a high magnetic field MRI in medium-sized $(3-10 \mathrm{~kg}$ ) or small (less than $1 \mathrm{~kg}$ ) animals [3-7], which, suggests that the sensitivity required for obtaining distinct MR images depends on the use of a strong magnetic field. However, a high magnetic field MRI unit possesses some disadvantages as compared with a low magnetic field unit, namely, the small space within the magnet available for the handling of subjects, difficult procedures for performing detailed clinical observations, high noise sound and running costs [7]. In contrast, these disadvantages are not present in the low magnetic field MRI, but little information is known about the appropriate basal technical conditions for its efficient use. The present study was therefore designed to establish the optimum technical conditions for images of small animals using a $0.2 \mathrm{~T}$ low field MRI unit, with a kitten as the subject.

A 3-week-old healthy male Japanese domestic kitten weighing $550 \mathrm{~g}$ and obtained from the Veterinary Teaching Hospital of Obihiro University of Agriculture and Veterinary Medicine was used for this investigation. The animal was anesthetized intravenously with pentobarbital sodium $(25 \mathrm{mg} / \mathrm{kg})$, and then placed in the prone position inside a $0.2 \mathrm{~T}$ permanent magnetic unit (MRP-20 EX: Hitachi Medical Corporation, Chiba, Japan) equipped with a custom-made solenoid coil ( $50 \mathrm{~mm}$ in diameter, Hitachi Medical Corporation, Chiba, Japan). The maximum strength of the gradient coil for the slice selection was 10 gauss $/ \mathrm{cm}$. T1 and T2 weighted images were obtained with the spin echo pulse sequence.

The relaxation time was measured to find the optimum parameter for the brain [2], but not for the spinal cord because the volume of tissue was too small. In the present study, the T1 relaxation time was $373-416 \mathrm{msec}$ and T2 relaxation time was $80-96 \mathrm{msec}$. There was little difference in these values between ours and data by Miyahara $e t$ al. , in which 366-398 msec (T1) and 77-89 msec (T2) were optimal in dogs. The $\mathrm{T} 1$ relaxation time in the low magnetic field was reported to be shorter than that in the high magnetic field [1], because the relaxation time depends on the strength of the magnetic field. Therefore, we scanned using the optimum repetition time (TR) and echo delay time (TE) in order to obtained the optimum parameter in the cat. As a result, a high-quality T1 weighted image was obtained when TR/TE was 500/25 msec, and an excellent $\mathrm{T} 2$ weighted image was produced at $1,800 / 110 \mathrm{msec}$ in a single slice technique. The parameters were different from the recent data by Miyahara et al.; 500/25 msec (T1 weighted image) and $2,000 / 100 \mathrm{msec}$ (T2 weighted image) in dogs. These species differences may be associated with differences in the development of the nerve cells or with proton density in the brain [1]. On the basis of these data, we decided to use the conditions $500 / 38 \mathrm{msec}$ for a multi-slice T1 weighted image, and $1,800 / 110 \mathrm{msec}$ for a multi-slice T2 weighted image as the optimum parameters for the head of the cat. The TE was changed considering the hardware conditions.

In the brain, transverse $\mathrm{T} 1$ weighted images [TR/ $\mathrm{TE}=500 / 38 \mathrm{msec}, 120 \mathrm{~mm}$ field of view (FOV), $5 \mathrm{~mm}$ slice thickness, $256 \times 256$ matrix] and $\mathrm{T} 2$ weighted images (TR/TE $=1,800 / 110 \mathrm{msec}, 120 \mathrm{~mm}$ FOV, $5 \mathrm{~mm}$ slice thickness, $256 \times 256$ matrix) revealed a definite contrast between the white and gray matter of the cerebrum, and the cerebral ventricles and the sulci were clearly distinguished (Fig. 1). The white-black contrast of most tissues of the cerebrum was reversed between $\mathrm{T} 1$ and $\mathrm{T} 2$ weighted images. Since the animal was so young, the brain contained more water and less myelin than in an adult animal. Thus, the relaxation time is considered to be slightly longer than in adult cats [1].

In sagittal $\mathrm{T} 2$ weighted images of the spinal cord $(\mathrm{TR} / \mathrm{TE}=1,500 / 110 \mathrm{msec}, 260 \mathrm{~mm}$ FOV, $5 \mathrm{~mm}$ slice thickness, $256 \times 256$ matrix) (Fig. 2), the intervertebral disks and cerebrospinal fluid showed a high signal intensity due to the presence of many protons, and the bodies of the vertebrae showed a low signal intensity, these being fewer protons.

MR images were obtained with a FOV of $120 \mathrm{~mm}$ and a matrix of $256 \times 256$, and so resolution of the images was less than $0.5 \mathrm{~mm}$. A $5 \mathrm{~mm}$ slice was considered to be somewhat thick for enough sensitivity, and for the partial volume effect to be excluded, but it is hoped that these problems will soon be solved as progress is made in 


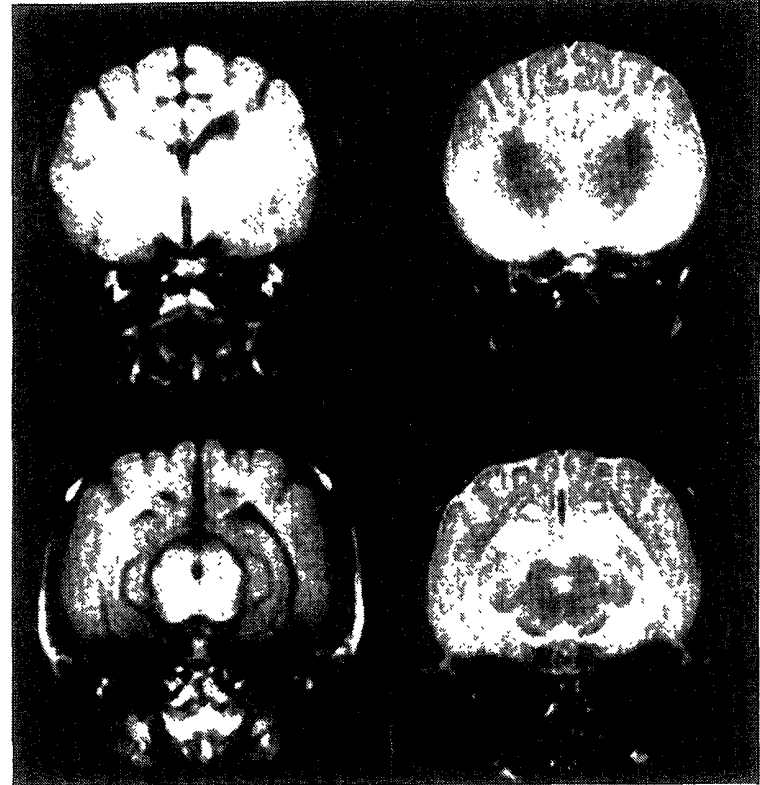

Fig. 1. Magnetic resonance imaging of the kitten's brain. (Transverse sections. Left side: T1 weighted images, TR $500 \mathrm{msec}$, TE 38-msec; right side: $\mathrm{T} 2$ weighted images, TR $1,800 \mathrm{msec}$, TE $110 \mathrm{msec}$; slice thickness, $5 \mathrm{~mm}$; FOV, 120 $\mathrm{mm}$; matrix, $256 \times 256$ ).

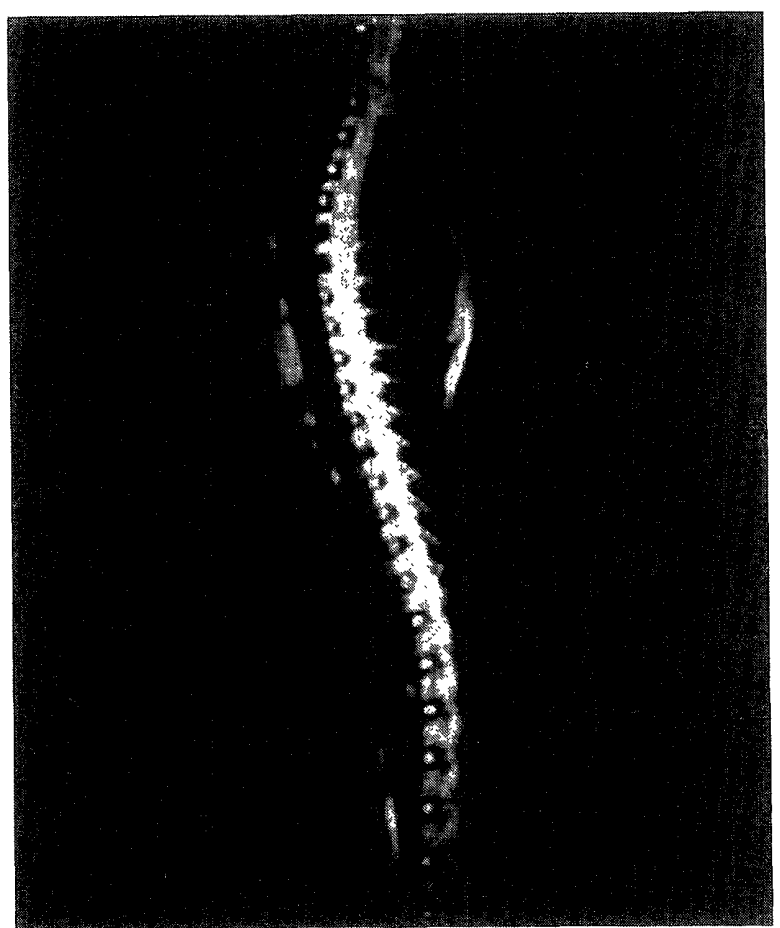

Fig. 2. Magnetic resonance imaging ( $\mathrm{T} 2$ weighted imaging) of the kitten's spine. (Sagittal section. TR 1,500 msec, TE $110 \mathrm{msec}$; slice thickness, $5 \mathrm{~mm}$; FOV, $260 \mathrm{~mm}$; matrix, 256 $\times 256)$. software and hardware.

In conclusion, the low magnetic field MRI with a suitable custom-made solenoid coil produced anatomical images of a small kitten noninvasively. It is generally said that low magnetic field MRI is difficult in small animals because of its poor sensitivity, but its scan times are almost the same as those for high magnetic field MRI. Moreover, when a suitable RF coil and optimum parameters are selected, the quality of the images produced is as good as that obtained with high magnetic field MRI. Therefore, low magnetic field MRI is not necessarily inferior to high magnetic field MRI, is useful for diagnoses of the CNS such as herniated disks and spinal disease in very young and small animals in the veterinary field, and may also have potential for protracted studies in experimental animals [7].

\section{REFERENCES}

1. Bottomley, P. A., Foster, T. H., Argersinger, R. E., and Pfeifer, L. M. 1984. Med. Phys. 11: 425-448.

2. Bottomley, P. A., Hardy, C. J., Argersinger, R. E., and Allen-Moore, G. 1987. Med. Phys. 14: 1-37.

3. Nakakoshi, T., Fujita, N., Johg-Hog, K., Takeichi, N., and Miyasaka, K. 1993. Invest. Radiol. 28: 1010-1014.

4. Ohashi, F., Kotani, T., Onishi, T., Katamoto, H., Nakata, E., and Friz-Zieroth, B. 1993. J. Vet. Med. Sci. 55: 875-876.

5. Sumi, M., Takahashi, M., Nagahiro, S., Korogi, Y., Sakamoto, Y., Nishimura, R., Hamatake, S., and Ushio, Y. 1993. Invest. Radiol. 28: 994-1003.

6. Tateno, Y. and Yamada, K. 1993. The Atlas of MRI and $\mathrm{CT}$ in Cats and Dogs. University of Tokyo Press, Tokyo.

7. Wolf, R. F. E., Lam, K. H., Mooyaart, E. L., Bleichrodt, R. P., Nieuwenhuis, P., and Schakenrad, J. M. 1992. Lab. Anim. 26: 222-227. 